\title{
Attitudes to reproductive genetic testing in women who had a positive BRCA test before having children: a qualitative analysis
}

\author{
Elizabeth Ormondroyd ${ }^{\star, 1}$, Louise Donnelly ${ }^{2}$, Clare Moynihan ${ }^{3}$, Cornelie Savona ${ }^{4}$, Elizabeth Bancroft ${ }^{5}$, \\ D Gareth Evans ${ }^{6}$, Rosalind A Eeles ${ }^{7}$, Stuart Lavery ${ }^{8}$ and Maggie Watson ${ }^{9}$
}

The scope of conditions for which preimplantation genetic diagnosis (PGD) is licensed has recently been expanded in the United Kingdom to include genetic predisposition to adult-onset cancer. This qualitative interview study explores reproductive decision making, knowledge of and attitudes to reproductive genetic testing (prenatal diagnosis and PGD) with 25 women aged 18-45 years who received a positive BRCA test in the United Kingdom before having children. In this cohort of younger women, $B R C A$ testing was motivated by risk management decisions; for some, $B R C A$ status has affected their later decisions about having children. The perceived severity of hereditary breast/ovarian cancer (HBOC) influences thoughts about passing on the mutation to children and willingness to consider reproductive genetic testing, but most participants do not believe HBOC is a condition for which pregnancy termination is justified. PGD is considered more acceptable and advantageous because it would prevent transmission to future generations, but women have concerns about selecting embryos and the fact that they and affected family members would not have been selected. Women would also be deterred by the need to undergo in vitro fertilisation (IVF) and ovarian stimulation for PGD. Awareness of reproductive testing options was very variable among the cohort. The findings highlight the complexities of reproductive decision making for young women who knowingly carry a BRCA mutation, and the dilemmas inherent to reproductive genetic testing when the condition being tested for also affects a prospective parent. Counselling and psychological support for BRCA-positive women and couples concerning reproductive options are strongly indicated.

European Journal of Human Genetics (2012) 20, 4-10; doi:10.1038/ejhg.2011.146; published online 3 August 2011

Keywords: $B R C A$; reproductive; attitudes; cancer; psychosocial; qualitative

\section{INTRODUCTION}

In 2006, following public consultation, The Human Fertilisation and Embryology Authority (HFEA; the independent regulator overseeing the use of gametes and embryos in fertility treatment and research in the United Kingdom) decided to expand the range of conditions for which it grants preimplantation genetic diagnosis (PGD) licences to include genetic predisposition to cancer, ${ }^{1}$ such as hereditary breast/ ovarian cancer (HBOC) caused by BRCA1 or BRCA2 gene mutations. PGD is a technically demanding procedure in which women undergo controlled ovarian stimulation to produce multiple eggs, which are recovered and fertilised in vitro. Embryos are cultured in the laboratory and biopsied at the eight cell stage. One cell is removed from each embryo and genetic analysis performed on these single embryonic cells, and selected embryos can then be transferred into the uterus. PGD may involve considerable physical and psychological stresses. ${ }^{2}$ Chances of a successful pregnancy following PGD do not exceed 50\% in any cycle. In the United Kingdom, the cost per cycle is between $£ 8000$ and $£ 11000$, although funding may be available on the
National Health Service. Female BRCA mutation carriers may have the additional risk of developing cancer because of controlled ovarian stimulation, although this is not yet quantified. ${ }^{3}$ Aromatase inhibitors have been used to reduce oestradiol levels in women with a history of breast cancer undergoing ovarian stimulation. ${ }^{4}$

Prior to the HFEA decision, conditions for which PGD had been licensed were highly penetrant (close to $100 \%$ of gene carriers affected), had onset in childhood or were ones for which treatment/prevention options are limited. The lifetime risk of breast cancer for female BRCA1/2 carriers is up to $85 \%$, and $27-60 \%$ of developing ovarian cancer, ${ }^{5}$ population-based estimates are somewhat lower. ${ }^{6}$ There is a higher risk of developing breast cancer at a younger age, and of bilateral cancer; onset before 25 years is unusual but does occur. ${ }^{7}$ The lifetime risks to male BRCA2 carriers of breast and prostate cancer are substantially higher than population risk, ${ }^{8}$ but for BRCAl only slightly different from population risks. ${ }^{9}$ Risk-reducing surgery (mastectomy, salpingo-oophorectomy) does not eliminate risk, ${ }^{10}$ and is not without possible compromise to quality of life and psychological well-being. ${ }^{11}$

\footnotetext{
${ }^{1}$ Department of Cardiovascular Medicine, John Radcliffe Hospital, Oxford, UK; ${ }^{2}$ Nightingale Centre and Genesis Prevention Centre, Wythenshawe Hospital, Manchester, UK; ${ }^{3}$ Psychology Research Group, The Institute of Cancer Research, Sutton, UK; ${ }^{4}$ South London and Maudsley NHS Foundation Trust, Bethlem Royal Hospital, Beckenham, UK; ${ }^{5}$ Department of Cancer Genetics, The Royal Marsden NHS Foundation Trust, Sutton, UK; ${ }^{6}$ Department of Genetic Medicine, St Mary's Hospital, Manchester, UK; ${ }^{7}$ Department of Cancer Genetics, The Institute of Cancer Research/The Royal Marsden NHS Foundation Trust, Sutton, UK; ${ }^{8}$ Department of Reproductive Medicine, Hammersmith Hospital, London, UK; ${ }^{9}$ Psychology Research Group, The Institute of Cancer Research/The Royal Marsden NHS Foundation Trust, Sutton, UK

*Correspondence: Dr E Ormondroyd, Department of Cardiovascular Medicine, Level 6 West Wing, John Radcliffe Hospital, Headington, Oxford OX3 9DU, UK Tel: +44 186523 4676; Fax: +44 186523 4677; E-mail: liz.ormondroyd@cardiov.ox.ac.uk
}

Received 26 January 2011; revised 25 May 2011; accepted 28 June 2011; published online 3 August 2011 
Prenatal diagnosis (PND) followed by termination of a pregnancy (TOP) carrier fetus ${ }^{12}$ is an alternative for known BRCA mutation carriers who want biological children not carrying the mutation. Concerns have been raised that PND for (breast) cancer predisposition represents a perversion of the original intention to protect people from the fate of an early, painful death or severe disability, and falls within the realm of deciding the 'desirability' of a particular life. ${ }^{13}$ Of the 26 $B R C A 1 / 2$ mutation carriers in the Netherlands, none viewed TOP for $B R C A$ as acceptable. ${ }^{14}$ In the United Kingdom, PND/TOP is not regulated by the HFEA (or other than by the Abortion Act 1967) and figures for its use for $B R C A$ are not available; anecdotally, it is extremely rare. Counselling for $\mathrm{PND} / \mathrm{TOP}$ includes discussion of the ethical implications of undergoing PND and not terminating a carrier pregnancy, which centre around loss of the child's autonomy in making future decision about presymptomatic testing. ${ }^{15}$ Earlier versions of the HFEA Code of Practice (sixth edition, 2003) stated: 'Indications for the use of PGD are expected to be consistent with current practice in the use of (post-implantation) prenatal diagnosis (PND), ${ }^{16}$ This ethical premise is supported by findings among health professionals in France ${ }^{17}$ that there is no difference in acceptability of one procedure compared with the other with respect to cancer predisposition. The clause was removed from later editions of the Code of Practice. ${ }^{18}$

Ethical objections to PGD have resulted in a wide variation in acceptability and regulation in developed countries. ${ }^{19,20}$ At issue is the conflict between parental autonomy in reproductive decision making and the welfare of embryos, children and society as a whole. ${ }^{21}$ PGD invokes fundamental questions including what characteristics convey the right to be born, and who should decide? ${ }^{22}$ PGD for cancer predisposition divides opinion among health professionals in Europe $^{17,23}$ and the United States. ${ }^{24,25}$ Attitudes among people at risk of cancer towards PGD are similarly mixed. ${ }^{26-28}$ However, all of these studies have been conducted with women of all age groups - for the majority of respondents, age may preclude having (further) children, and some studies include women who do not have a $B R C A$ mutation and so would not be able to use PGD.

This qualitative study sought to explore whether reproductive decision making has been affected by knowledge of $B R C A$ genetic status, whether participants have concerns about transmitting the mutation and if so, what are their attitudes towards available reproductive testing measures and contributors to these attitudes. Recruitment strategy focussed on women and men who had had BRCA genetic testing while childless and were $<45$ years old with the aim of uncovering attitudes and beliefs in people for whom reproductive testing is an option.

\section{METHODS}

\section{Participants}

The study was approved by the Royal Marsden NHS Foundation Trust Ethics Committee.

Eligible participants were women and men aged 18-45 years who tested positive in the preceding 5 years for a pathogenic BRCA1 or BRCA2 mutation and at the time of testing did not have children. Participants were recruited at two UK hospitals (Royal Marsden, and St Mary's Hospitals) between November 2007 and March 2010. Participants may have had a personal history of cancer, but were not within 2 years of diagnosis. Eligible people were considered by their clinical team to have no serious mental health contraindications, and were invited more than 6 months after their BRCA test result. Participant information sheets carried a brief description of PND and PGD. A total of 59 eligible people were identified and invited to participate: 55 women and 4 men.

\section{Data collection and analysis}

A qualitative approach was appropriate for exploring these complex sensitive issues. Data were collected using semistructured interviews at the participants' homes or a location of their choosing; several preferred to come to the hospital. Interviews lasted between 30 and $120 \mathrm{~min}$ and were guided by a set of openended topics devised from a review of relevant literature:

- Motivation for having a genetic (BRCA) test

- Reactions and accommodation to receiving a positive result

- Effects on reproductive decision making

- Knowledge of and attitude towards prenatal testing

- Knowledge of and attitude towards PGD

- Relative acceptability of PND/PGD

The interviewer pointed out that PGD involves in vitro fertilisation (IVF), which requires women to take ovarian-stimulating hormones. Each interview was audio recorded and transcribed verbatim. A thematic analysis of the transcripts was conducted according to an inductive theoretical framework. ${ }^{29}$ Inductive research is open-ended, exploratory and data driven, ${ }^{30}$ that is, not subject to a pre-existing coding frame. This framework focusses on prioritising participants' subjective accounts of their experience and of what is important to them. Themes were coded inductively to construct theory based on an understanding of how known cancer risk influences decision making with regard to having children. A subgroup of the team (EO, LD, CM and MW) met to define major thematic categories, including similarities and differences in participants' experiences and opinions, in a selected sample of interview transcripts, which provided a framework for systematic and iterative analysis of the other interviews by EO and LD. Emerging themes were compared across transcripts to test validity. No new themes emerged during the final interviews, suggesting that saturation was achieved.

\section{RESULTS}

Participant characteristics are shown in Table 1. In all, 25 women (55 invited) and 0 men participated (4 invited). Several women had

Table 1 Participant demographics

\begin{tabular}{|c|c|c|}
\hline & RMH participants (\%) & Manchester participants (\%) \\
\hline \multicolumn{3}{|l|}{ Age } \\
\hline $18-25$ & 0 & 0 \\
\hline $26-30$ & $4(27)$ & $4(40)$ \\
\hline $31-35$ & $3(20)$ & $3(30)$ \\
\hline $36-40$ & $5(33)$ & $1(10)$ \\
\hline $41-45$ & $3(20)$ & $2(20)$ \\
\hline Total & 15 & 10 \\
\hline \multicolumn{3}{|l|}{ Sex } \\
\hline $\mathrm{F}$ & $15(100)$ & $10(100)$ \\
\hline M & 0 & 0 \\
\hline \multicolumn{3}{|l|}{ Surgery } \\
\hline Mastectomya & $8(53)$ & $7(70)$ \\
\hline Oophorectomy ${ }^{b}$ & 0 & $2(20)$ \\
\hline None & $7(46)$ & $1(10)$ \\
\hline Cancer diagnosis & $4(27)$ & $2(20)$ \\
\hline \multicolumn{3}{|c|}{ Number of children } \\
\hline 0 & $10(66)$ & $7(70)$ \\
\hline 1 & $4(27)$ & $2(20)$ \\
\hline 2 & $1(6)$ & $1(10)$ \\
\hline \multicolumn{3}{|l|}{ Ethnic background } \\
\hline White, British & $10(66.6)$ & $10(100)$ \\
\hline White, Other & $5(33.3)$ & 0 \\
\hline
\end{tabular}

ancludes treatment/prophylactic

bProphylactic. 
had one or two children since BRCA testing; none had used PGD. One participant had used PND and continued with a noncarrier pregnancy. In the anonymised quotes, participants are coded 'R' (RMH patients) or ' $\mathrm{M}$ ' (Manchester patients).

\section{Impact of $B R C A$ result on thoughts about having children}

Among this cohort of women who have undergone genetic testing at a young age, the impact of cancer emerged strongly. For many, the development of cancer (if they did not undergo risk-reducing surgery) was seen as inevitable, and the decision to have genetic testing was primarily motivated by risk management strategies rather than thoughts about future children. A positive diagnostic BRCA genetic test may have an impact that is distinct from a cancer diagnosis, appearing to focus attention on the implications for existing family members and influencing thoughts about family planning. For participants R18 and R08, both with a personal history of cancer in their 20 s, their genetic test result changed their thinking about having children - R18 had terminated an earlier pregnancy because her understanding was that having a child increased the risk of recurrence:

'My cancer, in my head, was gone, so I was fine I would make sure it didn't come back, but now the cancer gene sits here every single day and I can't do anything with it ... (having a child would) increase my risk but it's not even comparable to the gene risk ...'

(R18, age 44, no children)

R08 had planned to get pregnant after her 5-year treatment with Tamoxifen, but while trying for pregnancy her genetic test result became available:

'You want to get pregnant and that's your end result ... so it's probably worked out well knowing at that time that I've got the gene, cause it does make you stop and think really, what are we doing? OK we want this but ... you do get a bit lost for a little while'.

(R08, age 36, no children)

This group had a heightened awareness of how illness in general can be hereditary, and are aware that their predisposition to cancer is known but may not be the only condition to run in the family, as M35 describes:

'if you've got that gene, god what other gene might you have that they don't know about at the moment'.

(M35, age 43, no children)

This appeared to be a mechanism for putting $B R C A$ status into perspective but influenced thinking about PGD; selecting embryos solely on the basis of their not carrying the BRCA mutation was considered questionable:

'You know you wipe that board clean, who do you get, you get rid of the embryos with the gene then you get a child with a completely different problem ... I almost want to say 'serves you right for messing around with this baby'

(R20, age 28, no children)

Perceived severity of HBOC in the context of reproductive decisions The perceived severity of breast/ovarian cancer predisposition, and the extent to which it is considered 'manageable', varied and was strongly influenced by personal experience. Decisions about whether to have children, whether HBOC justified TOP following prenatal testing, and opinions about PGD for HBOC were discussed in this context. Among the cohort were women who believed that HBOC is a relatively mild, manageable condition, to those who were inclined not to have children because they felt unwilling to pass on the BRCA gene. Several women had had children knowing their BRCA status and justified this in terms of their own and relatives' experience, such as R13:

'I definitely didn't think, I've got the gene, I wouldn't have children because I suppose I just look at my mother and me and it just seemed, it's something you can manage ... there was no way I would have said I wouldn't have children because of this gene'.

(R13, age 34, one child)

The perception that HBOC is manageable was not unique to women who have not had a cancer diagnosis, as R25 explains:

'I think I coped very well, I was never pessimistic or thinking I was going to die, but also it was one of the easiest courses of treatment to have?

(R25, age 41, no children)

Many women mentioned their belief that medical science would provide improvements in prevention and/or treatment options in the context of perceived severity.

In considering whether to have children, some participants also note that the risk of passing on the gene is 'only' 50\%, and that the risk of cancer is not $100 \%$ (although this is in contradiction to the perceived inevitability of cancer discussed earlier). This was clearly related to the availability of measures to reduce risk that many of these women had taken and/or planned, as R14 says:

'No, definitely no (to TOP for BRCA). It's just that they've got to take precautions against it, so to me it's just not major. It's not like saying 'there I've given you cancer' is it? There's only a percentage chance'.

(R14, age 28, one child)

The relative magnitude of risk led some participants to hope that a potential child would be a boy, or to feel less worried about existing male children.

The majority of this cohort was of the opinion that HBOC does not justify pregnancy termination, although they were tolerant of others who might make this decision, recognising that this is a very individual decision that might be influenced by personal and family experience and attitude to cancer risk:

'I wouldn't, ... if somebody decided to have prenatal testing during the pregnancy and said we're gonna end the pregnancy (long pause) I guess I could relate to that from seeing and talking about, you know the feelings I've seen my mother and sister go through'.

(R03, age 37, no children)

This contrasts with attitudes among the cohort towards TOP in general. All the women took a pro-choice stance while recognising that abortion is never an 'easy' decision; all could envisage personally terminating a pregnancy for disability, even if criteria for judging 'seriousness' differed. This was a personal assessment taking into 
account perceived quality of life and ability to cope. In the contexts of willingness to have a child at risk of HBOC and attitudes to TOP for $B R C A$, the participants compared disability or other medical conditions favourably with HBOC:

'a cousin's girlfriend has had a kidney transplant; it's not like I have to be on dialysis for the rest of my life, I've just had two mastectomies'.

(M35, age 43, no children)

'this isn't a gene for blindness or cystic fibrosis or some of the other awful genes which actually disable you from birth and have a massive impact on every aspect of your life; it doesn't disadvantage you, it might never disadvantage you'.

(R20, age 28, no children)

Some women voiced concerns about whether a potential child would 'blame' their mother for knowingly having a child at risk; reluctance to pass on the mutation might cause some to feel an obligation not to continue a pregnancy with the mutation, but recognise that termination would be very difficult to do in practice:

'we discussed it [PND and TOP for BRCA] and then you know I decided that we wouldn't... I've always wanted to be a mother so it's ... but looking at somebody else going through it I can see it's probably the best thing to terminate a pregnancy. I suppose I'm looking at someone else making a decision without emotions and you think, why would you want to impose cancer on somebody else?'

(R08, age 36, no children)

R15 is the only participant to have actually used PND for BRCA. She developed cancer in her early 20s, without, at the time of her diagnosis, a family history of cancer. A large part of the interview concerned the major impact that her diagnosis and treatment had; part of her life plan was to have children young, and this was thwarted by her cancer treatment that included 5 years of taking Tamoxifen. She empathises with her mother's view that watching a daughter suffer cancer is worse than having it personally, and, on receiving her positive $B R C A$ result at the age of 25 , thought:

'This has been impossible, there's no way I'm gonna put my child through that, there's no way'

At the time, she investigated the option of PGD, not then licensed in the United Kingdom, and hence chose PND, unaware of the dilemma that would pose:

'I got pregnant and at that time my whole thinking was, I was gonna have a CVS at 3 months, check if the baby had the (BRCA) gene, and if the baby had the gene I probably wouldn't go through with the pregnancy ... from the minute I saw the baby's heart beat, you know two things 'I don't want a baby with the cancer gene but I'm not giving up this baby'.

(R15, age 32, one child)

\section{Value of life at risk of $\mathrm{HBOC}$}

There are two aspects to this theme, which describes participants' reflections on reproductive selection (which may include simply not having children) based on the BRCA gene. The first concerns the value of existing lives that would not have existed if the reproductive options under discussion had been available. R07 recounted a discussion she had been part of at a support group for carriers of the BRCA mutation:

'everyone at the table asked the same question to each other ... we agreed that if (earlier generations) had decided not to have children then none of us would be there. That was a kind of powerful idea and I think we all wanted to be there'.

(R07, age 31, no children)

Some considered that if they were to have children they have a responsibility to use reproductive technology, but found it difficult to reconcile this with their view that existing individuals would not have been born:

'other days when you just think, I have the gene, my dad has the gene, my auntie, my grandma, all of them could have been discarded'

(R20, age 28, no children)

In contrast, for others, the significance of cancer is so great that not having existed would have been a price worth paying - R18 relates this to her thoughts about passing on the gene:

'... I do feel that my whole life is cancer, and actually I could cry now, it's awful to think you might not (have been born) but then I wouldn't know the difference ... with the knowledge that I have I don't know that I would want my child to have the aimless worry that I feel I have'.

(R18, age 44, no children)

The second aspect of this theme concerns the relative morality of preand post-implantation selection. Participants were not in general concerned with morality in the sense of relative right to life; where moral differences were recognised between a pre-implantation embryo and a fetus of several weeks of gestation, they were expressed subjectively or from a practical point of view:

'I think, for me, if you're already pregnant then you know there is something growing inside you ... an embryo in its own right can't be called a living being, can it?'

(R03, age 37, no children)

'the embryo screening is more positive, you're getting something positive you're not taking something away ... I would choose the embryo screening'

(M35, age 43, no children)

$\mathrm{PND} / \mathrm{TOP}$ was seen as more emotionally and/or practically traumatic, particularly because of the $50 \%$ chance with each pregnancy. Discarding embryos was not seen as morally problematic, as R16 says:

'I mean, so it was this egg that got fertilised instead of that one, well so what? It's only with the benefit of hindsight that you even think about it'.

(R16, age 37, no children)

Participants prefer the option of PGD over PND/TOP, at least in theory. $\mathrm{PND} / \mathrm{TOP}$ is seen as more personal, a judgement made on the value of a specific life that is, like theirs, considered very much worth living, as R20 says:

'I couldn't make that decision (PND/TOP) based on something I've got'.

(R20, age 28, no children) 
In contrast, PGD is less personal; it would not only reduce risk to an individual child, but is also a means to stop transmission of this gene that has caused havoc, as R14 says:

'It's also the passing it down, it would just go on forever, whereas I think if you eliminate it and it starts stopping then there's gonna be a lot less people losing their mums at young ages like I did ...'

(R14, age 28 , one child)

Supporting this interpretation, the women were unanimous that if they were to use PGD, the gender of implanted embryos would be irrelevant - the goal would be to prevent transmission of the gene rather than reduce risk to an individual child, as R03 says:

'I can't see why you would treat them any differently, because ultimately, it might not be such a high risk for a boy but it's just the thought that that gene would be carrying on down the line ... I don't want to pass this on at all.'

(R03, age 37, no children)

\section{Practical considerations}

Although the evidence does not clearly contraindicate IVF in women with BRCA mutations, many would be reluctant to consider it, either because of friends' experience of undergoing IVF or an aversion to taking additional hormones that might increase their risk of developing cancer. A commonly expressed view was that it would be undesirable to achieve a pregnancy after PGD and then personally to develop cancer that the child would witness and possibly result in the loss of a parent:

'it's more important for me to be alive and to be still living rather than jeopardise it by having something that could potentially cause the cancer to come back, because I've got my family as well, not just my son ... I wouldn't have gone for IVF, no.

(M31, age 42, one child)

Some felt that PGD would be more acceptable if a woman had coexisting fertility problems that might indicate IVF anyway, but R08, who has tried without success to achieve a pregnancy 'naturally', rules out IVF - she relates this directly to her genetic status as opposed to her personal history of cancer:

'going through IVF is a problem, it's so personal to my body, we decided not to go through that knowing now that I've got the gene... giving my body hormones is not a good thing for me personally'.

(R08, age 36, no children)

For other participants, relationship issues, availability of PGD and/or timing were seen as barriers to the use of PGD. Age is also a factor, as R25 says:

'I just want to have a child but if I were 25, it'd be much fairer to the child to have (PGD) ... but for a woman over 40 if the choice were between having a child in vitro but with very small chances of success and having a foetus with the gene I would go for natural conception'.

(R25, age 41, no children)

Awareness of reproductive options and counselling/support needs For some, awareness of reproductive options was very limited. For those who were aware, most had heard about it through the media rather than from health professionals. Two women were exceptional in having sought information about PGD. One highlights a perceived gap in counselling provision for PGD; having 'battled' to get a referral, she described her PGD consultation:

'She [doctor] was definitely under the impression that we'd made a decision but it was actually just so we could learn about the options ... she had a power point that she ran through on her laptop and explained the process but I don't think she was geared up to be talking to someone for the first time.

(R20, age 28, no children)

This suggests that although factual information is required, there is a need for women's psychological needs to be accommodated, with the opportunity for them and their partners to raise and discuss these issues during consultations.

These particularly well-informed women perhaps predict increased future interest in PGD for $B R C A$, and as such their contributions are especially informative. In contrast to the apparently less well-informed women, who felt motivated to consider PGD were it not for the requirement to undergo hormone stimulation, R15 and R20 spent significant time deliberating this option, and over time developed their thinking about some of the barriers presented here. Both women have been significantly affected by cancer, and are strongly motivated to do whatever they can to control the risk, both to themselves and their future children. They recognise that the decision to use reproductive technologies is far more complex than the personal risk management decisions they have taken, to such an extent that they are paralysed by an inability to move forward.

' ... I found myself thinking, I'm testing her for this how do you know the baby they pick out is not gonna have something else? Am I being too obsessive about this? The difference between having the CVS and PGD is, I got pregnant naturally so this is the baby that was intended to be ... that's what made me say, look, I don't think $P G D$ is for me'.

(R15, age 32, one child)

'... do you get to go and point and say 'I'll have that one and that one?' ... I just see this can of worms that ultimately has got to be opened. I never imagined that having a family would involve this'.

(R20, age 28, no children)

\section{DISCUSSION}

Focussing on women of appropriate age, and who are making decisions about having children in the knowledge of positive BRCA status, this paper explores and contrasts attitudes and intentions towards reproductive testing options, provides explanations for the differences in attitudes towards PND/TOP and PGD, and highlights the need for psychosocial support in this area. Identified themes concern the impact of genetic testing before having children, the perceived severity of HBOC and how this relates to willingness to pass on the gene, and relative acceptability of reproductive options. Perceived severity is influenced by personal and family cancer history, current risk management options and faith in future advancements in medical science, and by how well women believe they coped or would cope with cancer. Most women value their own life and that of family members who also carry the gene mutation, and although some have concerns about passing on the mutation to children, they do not believe HBOC is a condition for which pregnancy termination is 
justified. This objection seems not to be based on the principles of morality; the women believe in the right to choose whether to continue a pregnancy on any grounds, and believe there are conditions more serious than HBOC for which they would terminate. Women consider PGD for BRCA to be acceptable in theory, although many are deterred by the need to undergo IVF and ovarian stimulation. The contrasting attitudes to PND/TOP and PGD are, we suggest, motivated by a wish to prevent transmission of the BRCA gene mutation, without passing judgement on a specific life, or fetus. The findings also highlight the complexities of reproductive decision making for women who have had BRCA genetic testing at this life stage, even after a cancer diagnosis, which may not have been fully appreciated before testing and have implications for counselling service provision.

The HFEA advocate that, 'When deciding if it is appropriate to provide PGD in particular cases, the seriousness of the condition in that case should be discussed between the people seeking treatment and the clinical team. ${ }^{18}$ As this study shows, 'seriousness' is an important factor in attitude towards having children at risk, but may be an oversimplification of the perceived imperative to avoid it. Women who have similar personal or family histories of cancer may have very different psychological responses and attitudes to risk management. Determinants of psychological adaptation may include previous risk perception, age at development of cancer, but personality and a perceived need to control risk may also be factors. If access to PGD is to be granted on a case-by-case basis, these factors need to be taken into account. There is a potential contradiction between the nonselection of carrier males for implantation and the licensing requirements for PGD given that males are not at high risk of cancer, and indeed for BRCA1 may have no extra risk.

Our findings compare with recent studies of attitudes towards $\mathrm{PGD}^{26-28}$ that suggest that high-risk women (with the caveat that some of these studies include non-BRCA carriers and women post reproductive age) express concerns about transmitting the BRCA mutation and are broadly in favour of PGD being offered for $B R C A$, although respondents feel underinformed about the process and procedures involved. ${ }^{31}$ Approximately half of a cohort ${ }^{32}$ of $B R C A$ high-risk women and men believe they would consider PND/TOP or PGD, with no appreciable difference in the numbers who would consider either. The difference between hypothetical and actual intent is an acknowledged limitation in these studies. The present study addressed this by focussing on mutation carriers who are of approximate reproductive age, and whose mutation status was known before they had children. We find that PGD is considered more acceptable than PND/TOP for BRCA, but not because of a perceived moral difference between the developmental status of an embryo versus an implanted fetus; rather, it represents an unwillingness to end a life on the basis of cancer predisposition, with which participants and other relatives also live.

Our data support the argument of Cameron and Williamson ${ }^{33}$ that 'ethical decisions (about PGD/PND) taken are far closer to an ad hoc, relativist, and utilitarian model than one based on any of religious codes, scientific knowledge, consistency in the view of the status of the embryo'. We suggest that this is linked to the perceived utility of PGD for later-onset conditions: to prevent transmission of the deleterious gene to future generations, without the need to terminate a particular life. This contrasts with PGD for more 'serious' conditions with earlier onset, where the desired outcome is simply to have a healthy child. ${ }^{34}$ If correct, it might be expected that PGD would be more acceptable than PND/TOP for other later-onset conditions, as reported for HD. ${ }^{35}$ Our finding that PGD is more acceptable to these women, notwithstanding the need to undergo IVF, is borne out by reports of referrals for PGD for BRCA in the United Kingdom. ${ }^{36}$ Further research on the experience of going through PGD for $B R C A$ is required.

Studies based in other countries have found that health professionals are divided over acceptability of cancer genetic testing in the reproductive arena. In France ${ }^{17}$ and Spain, ${ }^{32}$ approximately half of health professionals surveyed are of the opinion that PND/PGD should be offered for BRCA. In the United States, approximately half of gynaecological oncologists and obstetric/gynaecologists surveyed thought PGD appropriate for $B R C A ;<20 \%$ reported that they were 'knowledgeable' or 'very knowledgeable' about PGD. ${ }^{25}$ Interestingly, the proportions of surveyed health professionals who consider PGD acceptable are consistent with the proportions who view $\mathrm{PND} / \mathrm{TOP}$ as acceptable for the same conditions. In the United Kingdom, data from clinical genetics units are lacking. Staff working at an assisted conception unit have concerns about the appropriateness of offering PGD for cancer but consider that patients' opinions should generally override professional concerns. ${ }^{23}$

\section{Limitations}

Our recruitment strategy aimed to include men, but only a small number were eligible and they declined participation. Given our finding that women choose $B R C A$ testing in order to guide risk management decisions, men may be less motivated to test presymptomatically at a relatively young age. Most men having $B R C A$ testing do so when they have children, limiting the number available. A recent study suggests that a minority of men would consider PGD for BRCA. ${ }^{37}$ This requires further research. Response rate was $<50 \%$ among women, and hence the findings of this study may not reflect attitudes of all eligible women. The majority of our sample are of White British origin ethnicity, and although the RMH cohort included women of White non-British origin, it would be informative to explore these issues with a more ethnically diverse sample.

\section{Implications for clinical practice}

Women in our cohort had limited awareness of PND/TOP or of PGD for $B R C A$. Where they were aware, this was mostly as a result of media coverage, and a minority had actively sought information or referral for discussion of PGD. Up to now, genetic counselling regarding HBOC has concentrated on psychosocial adaptation and risk management. Our study shows that reproductive issues and options are very relevant and appropriate subjects for genetic counselling, particularly for younger carriers who have not begun or completed their families. The sensitivity and complexities of these issues, and the likelihood that they will require discussion with appropriately trained clinicians, may have presented barriers to raising them, but this situation is untenable in the light of the HFEA decision. Our results indicate a clear need for additional psychosocial support to help resolve these issues.

\section{CONFLICT OF INTEREST}

The authors declare no conflict of interest.

\section{ACKNOWLEDGEMENTS}

We are grateful to Dr Rachel Shaw for comments on the study design, Sue Davolls for support in preparing the funding application and to Ben Roberts for cross-checking/typing of some of the interview transcripts. We are especially grateful to those who kindly participated and gave their time so willingly. This research was supported by Cancer Research UK (Number C1226 A7920) and NIHR support to the Biomedical Research Centre at The Institute of Cancer Research and RMH. 


\section{AUTHOR CONTRIBUTIONS}

EO generated the study idea and contributed significantly to the funding application, interviewed most of the RMH cohort and jointly analysed all the data; $\mathrm{CM}$ assisted in writing the funding application and analysis of a subsample of the cohort interviews; DGE, RAE, and SL were the responsible clinicians and contributed to writing the funding application; LD interviewed the Manchester cohort and jointly analysed all the data; CS provided administrative support and transcribed the majority of the tapes; $\mathrm{EB}$ assisted with recruitment of participants and in obtaining background demographic information; MW as PI provided overall management of the project and assisted with aspects of the study including the funding application and data analysis. All authors contributed to writing the study report.

1 HFEA Choices and Boundaries -public discussion summary. Available at: http://www.hfea.gov.uk/docs/Choices_and_boundaries_Report_2006_summary.pdf.

2 Lavery SA, Aurell R, Turner C et al: Preimplantation genetic diagnosis: patients' experiences and attitudes. Hum Reprod 2002; 17: 2464-2467.

3 Venn A, Watson L, Bruinsma F et al: Risk of cancer after use of fertility drugs with in-vitro fertilisation. Lancet 1999; 354: 1586-1590.

4 Azim AA, Costantini-Ferrando M, Oktay KJ: Safety of fertility preservation by ovarian stimulation with letrozole and gonadotropins in patients with breast cancer: a prospective controlled study. Clin Oncol 2008; 26: 2630-2635.

5 Ford D, Easton DF, Stratton M et al: Genetic heterogeneity and penetrance analysis of the BRCA1 and BRCA2 genes in breast cancer families. The Breast Cancer Linkage Consortium. Am J Hum Genet 1998; 62: 676-689.

6 Antoniou A, Pharoah PDP, Narod S et al: Average risks of breast and ovarian cancer associated with mutations in BRCA1 or BRCA2 detected in case series unselected for family history: a combined analysis of 22 studies. Am J Hum Genet 2003; 72: 1117-1130.

7 Evans DG, Moran A, Hartley R et al: Long term outcomes of breast cancer in women aged 30 years or younger, based on family history, pathology and BRCA1/BRCA2/TP53 status. Br J Cancer 2010; 102: 1091-1098.

8 Thompson D, Easton D: Variation in cancer risks, by mutation position, in BRCA2 mutation carriers. Am J Hum Genet 2001; 68: 410-419.

9 Thompson D, Easton DF: Cancer Incidence in BRCA1 mutation carriers. J Nat/ Cancer Inst 2002; 94: 1358-1365.

10 Hartmann LC, Sellers TA, Schaid DJ et al: Efficacy of bilateral prophylactic mastectomy in BRCA1 and BRCA2 gene mutation carriers. J Natl Cancer Inst 2001; 93: 1633-1637.

11 Hopwood P, Lee A, Shenton A et al: Clinical follow-up after bilateral risk reducing ('prophylactic') mastectomy: mental health and body image outcomes. Psychooncology 2000; 9: 462-472.

12 Lancaster JM, Wiseman RW, Berchuck A: An inevitable dilemma: prenatal testing for mutations in the BRCA1 breast-ovarian cancer susceptibility gene. Obstet Gynecol 1996; 87: 306-309.

13 Wagner TM, Ahner R: Prenatal testing for late-onset diseases such as mutations in the breast cancer gene 1 (BRCA1). Just a choice or a step in the wrong direction? Hum Reprod 1998; 13: 1125-1126.

14 Lodder LN, Frets PG, Trijsburg RW et al: Attitudes towards termination of pregnancy in subjects who underwent presymptomatic testing for the BRCA1/BRCA2 gene mutation in the Netherlands. J Med Genet 2000; 37: 883-884.
15 Borry P, Evers-Kiebooms G, Cornel MC et al: Public and Professional Policy Committee (PPPC) of the European Society of Human Genetics (ESHG). Genetic testing in asymptomatic minors: background considerations towards ESHG Recommendations. Eur J Hum Genet 2009; 17: 711-719.

16 HFEA $6^{\text {th }}$ Code of Practice (2003); available at: http://www.hfea.gov.uk/docs/Code_of_ Practice_Sixth_Edition.pdf.

17 Julian-Reynier C, Chabal F, Frebourg T et al: Professionals assess the acceptability of preimplantation genetic diagnosis and prenatal diagnosis for managing inherited predisposition to cancer. J Clin Oncol 2009; 27: 4475-4480.

18 HFEA $8^{\text {th }}$ Code of Practice (2009); available at: http://www.hfea.gov.uk/docs/8th_Code of_Practice.pdf.

19 Knoppers BM, Isasi RM: Regulatory approaches to reproductive genetic testing. Hum Reprod 2004; 19: 2695-2701.

20 Turillazzi E, Fineschi V: Preimplantation genetic diagnosis: a step by step guide to recent Italian ethical and legislative troubles. J Med Ethics 2008; 34: e21.

21 Robertson JA: Extending preimplantation genetic diagnosis: medical and non-medical uses. J Med Ethics 2003; 29: 213-216.

22 Roberts C, Franklin S: Experiencing new forms of genetic choice: findings from an ethnographic study of preimplantation genetic diagnosis. Hum Fertil (Camb) 2004; 7: 285-293.

23 Williams C, Ehrich K, Farsides B, Scott R: Facilitating choice, framing choice: staff views on widening the scope of preimplantation genetic diagnosis in the UK. Soc Sci Med 2007; 65: 1094-1105.

24 Offit K, Kohut K, Clagett B et al: Cancer genetic testing and assisted reproduction. J Clin Oncol 2006; 24: 4775-4782.

25 Brandt AC, Tschirgi ML, Ready KJ et al: Knowledge, attitudes, and clinical experience of physicians regarding preimplantation genetic diagnosis for hereditary cancer predisposition syndromes. Fam Cancer 2010; 9: 479-487.

26 Menon U, Harper J, Sharma A et al: Views of BRCA gene mutation carriers on preimplantation genetic diagnosis as a reproductive option for hereditary breast and ovarian cancer. Hum Reprod 2007; 22: 1573-1577.

27 Staton AD, Kurian AW, Cobb $\mathrm{K}$ et al: Cancer risk reduction and reproductive concerns in female BRCA1/2 mutation carriers. Fam Cancer 2008; 7: 179-186.

28 Quinn G, Vadaparampil S, Wilson C et al: Attitudes of high-risk women toward preimplantation genetic diagnosis. Fertil Steril 2009; 91: 2361-2368.

29 Boyatzis RE: Transforming Qualitative Information: Thematic Analysis and Code Development. USA: Sage, 1998.

30 Braun V, Clarke V: Using thematic analysis in psychology. Qual Res Psychol 2006; 3: 77-101.

31 Quinn GP, Vadaparampil ST, Tollin S et al: BRCA carriers' thoughts on risk management in relation to preimplantation genetic diagnosis and childbearing: when too many choices are just as difficult as none. Fertil Steril 2010; 94: 2473-2475.

32 Fortuny D, Balmaña J, Graña B et al: Opinion about reproductive decision making among individuals undergoing BRCA1/2 genetic testing in a multicentre Spanish cohort. Hum Reprod 2009; 24: 1000-1006.

33 Cameron C, Williamson R: Is there an ethical difference between preimplantation genetic diagnosis and abortion? J Med Ethics 2003; 29: 90-92.

34 Franklin S, Roberts C: Born and Made: An Ethnography of Preimplantation Genetic Diagnosis. USA: Princeton University Press, 2006.

35 Delatycki MB: Commentary on Spriggs: genetically selected baby free of inherited predisposition to early onset Alzheimer's disease. J Med Ethics 2003; 29: 120.

36 SenGupta S, Fordham K, Mamas T et al: Preimplantation genetic diagnosis for BRCA 1/2. Poster Abstract, 10th International Congress on Preimplantation Genetic Diagnosis, May 2010, Montpellier, France.

37 Quinn GP, Vadaparampil ST, Miree CA et al: High risk men's perceptions of pre-implantation genetic diagnosis for hereditary breast and ovarian cancer. Hum Reprod 2010; 25: 2543-2550. 